\title{
Incidence and outcomes of eating disorders during the COVID-19 pandemic
}

\author{
Maxime Taquet, John R. Geddes, Sierra Luciano and Paul J. Harrison
}

\section{Summary}

There are concerns that eating disorders have become commoner during the coronavirus disease 2019 (COVID-19) pandemic. Using the electronic health records of 5.2 million people aged under 30 , mostly in the USA, we show that the diagnostic incidence was 15.3\% higher in 2020 overall compared with previous years (relative risk $1.15,95 \% \mathrm{Cl} 1.12-1.19$ ). The relative risk increased steadily from March 2020 onwards, exceeding 1.5 by the end of the year. The increase occurred solely in females, and primarily related to teenagers and anorexia nervosa. A higher proportion of patients with eating disorders in 2020 had suicidal ideation (hazard ratio HR $=1.30,1.16-1.47$ ) or attempted suicide (HR=1.69, 1.21-2.35).

\section{Keywords}

COVID-19; eating disorders; anorexia nervosa; epidemiology; eating disorders NOS.

\section{Copyright and usage}

(c) The Author(s), 2021. Published by Cambridge University Press on behalf of the Royal College of Psychiatrists. This is an Open Access article, distributed under the terms of the creative Commons Attribution licence (http://creativecommons.org/ licenses/by/4.0/), which permits unrestricted re-use, distribution, and reproduction in any medium, provided the original work is properly cited.
An increased incidence of eating disorders during the COVID-19 pandemic has been reported but not accurately or robustly quantified. ${ }^{1-3}$ Referrals for eating disorders have doubled in the UK. ${ }^{4}$ This study used electronic health records (EHR) to assess the relative incidence and outcomes (in terms of suicidal attempt, ideation and death) of eating disorders during the COVID-19 pandemic compared with previous years.

\section{Method}

We used TriNetX Analytics, a federated EHR network with anonymised data from 81 million patients, $93 \%$ of whom are in the USA. The participating healthcare organisations are a mixture of hospitals, primary care and specialist providers, and include insured and uninsured patients. For further details about TriNetX see Supplementary File (available at https://doi.org/10.1192/bjp. 2021.105) and Taquet et al. ${ }^{5}$

The incidence of a first diagnosis of eating disorder (ICD-10 code F50 $)^{6}$ was estimated for every 2-month period between 20 January 2020 (the date of the first recorded patient with COVID19 in the USA) and 19 January 2021 in patients under 30 years old who made at least one healthcare visit during this time. The incidence was compared with those in corresponding periods in 2019 using $\chi^{2}$-tests. Stratified results by age, gender and subcategories of eating disorder are provided. We also compared the 2020 data with 2017 and with 2018. In a sensitivity analysis, patients were only included if they also made a healthcare visit between 20 January 2021 and 28 April 2021 (or the same period in 2020 for comparison), thus guaranteeing that they were not lost to follow-up.

We assessed whether a diagnosis of COVID-19 (ICD-10 U07.1) was associated with a higher risk of subsequent eating disorders compared with a cohort of patients who had made a healthcare visit for another reason (matched for timing of the visit (in 2-month strata), demographics and risk factors for COVID19; see the Supplementary Methods).

The risk of suicide ideation, attempt and death within 6 months of an eating disorder diagnosis during the pandemic was compared with the previous 3 years using Kaplan-Meier analysis. For further details of analyses, see Supplementary Methods. STROBE reporting guidelines were followed.

\section{Results}

A total of 5186451 patients met the inclusion criteria $(55.3 \%$ female, $44.4 \%$ male, $0.3 \%$ other; mean age: 15.4 years, s.d. $=8.98$ ). There were 8471 people who were diagnosed with an eating disorder in the defined pandemic period $(78.1 \%$ female, mean age 16.2 years (s.d. $=7.2)$ and 19843 (75.4\% female; mean age 16.3 years (s.d. = 7.6)) during the prior 3 years. For details see Supplementary Table 1.

The incidence of a first diagnosis of an eating disorder has increased during the pandemic. An overall excess of $15.3 \%$ was observed compared with the previous year (relative risk $(R R)=1.15$, 95\% CI 1.12-1.19, $P<0.001$; Supplementary Table 2) with significant excesses observed in each 2-month period during the pandemic except for 20 March to 19 May 2020 (Fig. 1(a)). Similar results were found in the sensitivity analysis $(\mathrm{RR}=1.34,95 \% \mathrm{CI} 1.29-1.40$; with significant excess observed for all but the 20 March to 19 May 2021 period; $P<0.001$; Supplementary Fig. 1). Results were also similar if 2020 was compared with 2017 or 2018 (see Supplementary Table 2).

The increased risk of eating disorders during the pandemic period was limited to females (Fig. 1(b)), was greatest for 10- to 19-year-olds (Fig. 1(c)), and mostly affected anorexia nervosa diagnoses (Fig. 1(d)).

As shown in Supplementary Table 1 and Supplementary Fig. 2, compared with patients diagnosed with an eating disorder in the previous 3 years, those diagnosed during the pandemic were at a higher risk of attempting suicide $(0.89 \%$ (95\% CI $0.68-1.16) v$. $0.52 \%$ (95\% CI 0.43-0.64); hazard ratio (HR) $=1.69$ (95\% CI 1.21 $-2.35), P=0.0015)$ and having suicidal ideation $(6.3 \%(95 \% \mathrm{CI}$ $5.7-7.0)$ v. $4.8 \%$ (95\% CI $4.5-5.1) ; \mathrm{HR}=1.30$ (1.16-1.47), $P<$ $0.001)$, but death did not differ.

Patients diagnosed with COVID-19 were not at an increased risk of an eating disorder compared with a matched cohort of patients who made a visit to a healthcare organisation for a different reason during the pandemic (adequate matching was achieved for all covariates; Supplementary Tables 3-7; $\mathrm{HR}=0.86,95 \% \mathrm{CI}$ $0.65-1.12, P=0.26$, Supplementary Fig. 3). 
(a)

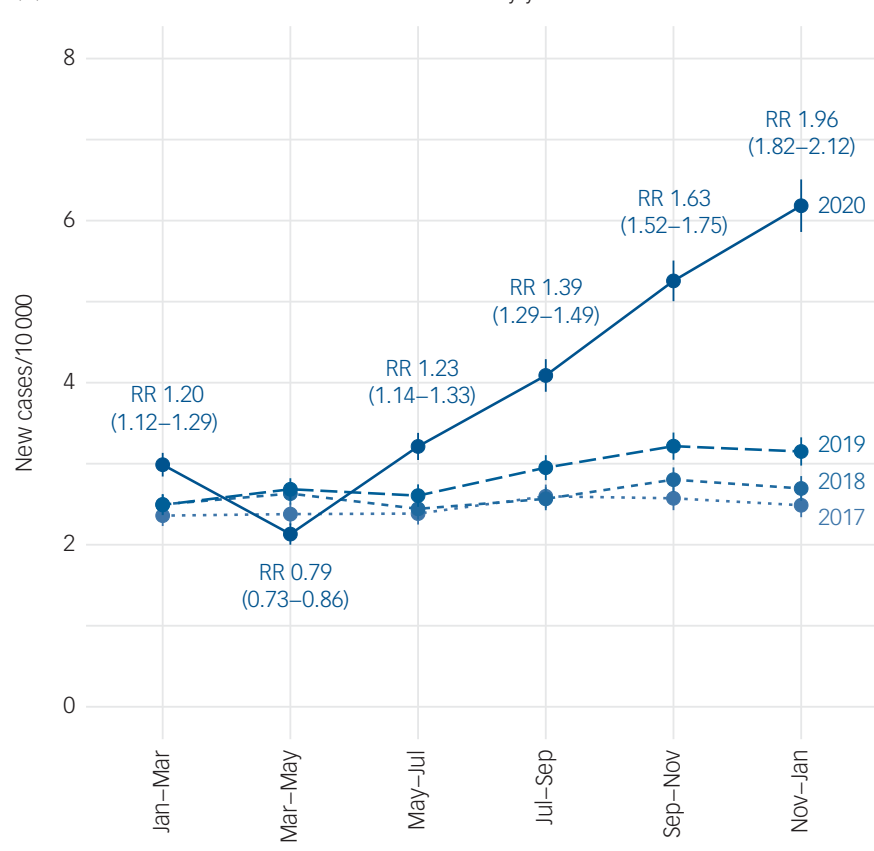

(b)

Incidence and relative risk (compared with 2019) by gender
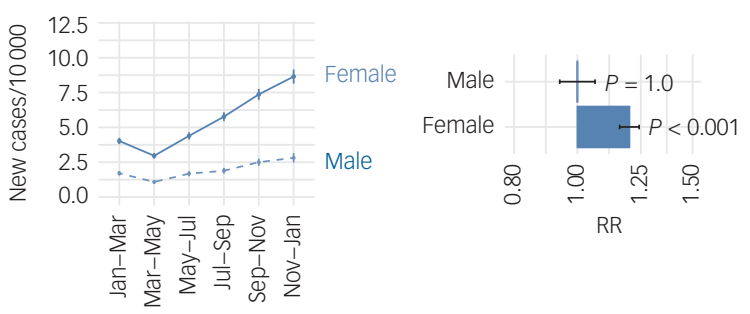

(C) Incidence and relative risk (compared with 2019) by age

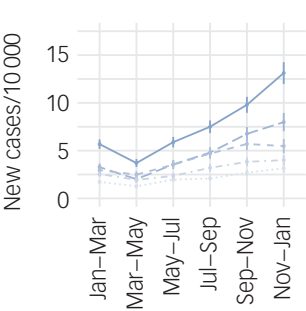

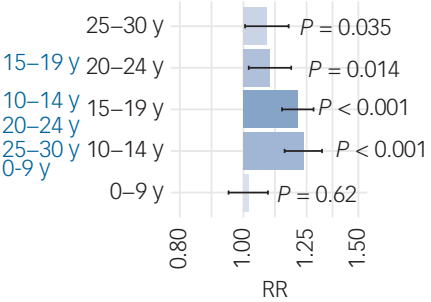

(d) Incidence and relative risk (compared with 2019) by disorder

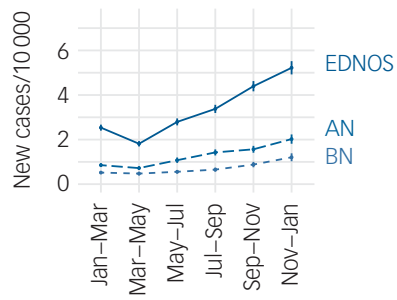

\section{Fig. 1 Incidence of eating disorders during versus before the COVID-19 pandemic.}

(a) Incidence of eating disorders in 2-monthly periods during the pandemic (20 January 2020 to 19 January 2021) compared with previous years (20 January 2017 to 20 January 2019 and 19 January 2018 to 19 January 2020). The relative risks (RR) and their $95 \%$ confidence intervals are provided for each 2-monthly period during the pandemic compared with the same period in 2019. (b) Incidence stratified by gender. (c) Incidence stratified by age group. (d) Incidence stratified by eating disorder subtype. RR in each stratum compared with the corresponding stratum in the previous year. RR > 1 indicates a risk that is higher during the pandemic. AN, anorexia nervosa; BN, bulimia nervosa; EDNOS, eating disorder not otherwise specified; $Y$, year.

\section{Discussion}

These data show an increased incidence of eating disorders during the COVID-19 pandemic, and with higher rates of suicidal behaviour among those diagnosed. After a decrease in the early part of 2020 (which likely reflects the marked reduction in all diagnoses made in the EHR network during this period ${ }^{7}$ ), the incidence of eating disorders increased steadily throughout the rest of 2020, such that the RR compared with previous years had exceeded 1.5 by the end of the year (being 1.96 in the primary analysis (Fig. 1 (a)), and 1.59 in the sensitivity analysis (Supplementary Fig. 1)). The mechanisms underlying these observations remain to be determined, although a range of plausible explanations have been proferred. ${ }^{2}$ The finding that the increase was seen only in females, and was greater in teenagers and for anorexia nervosa, may help focus future investigations. No increased risk was observed among people diagnosed with COVID-19 infection, unlike many other psychiatric disorders in this EHR network. ${ }^{5,7}$

The main strength of the study is the use of a large EHR network that permits robust and up-to-date estimates of incidence and RR. Limitations include those inherent to EHR studies (for example lack of information about the accuracy or completeness of diagnoses; see the Supplementary File and Taquet et $\mathrm{al}^{5}$ for discussion). Our findings relate to patients who made a healthcare visit and received a diagnosis, and should not be generalised to the wider population, especially as many patients with eating disorders do not seek treatment. ${ }^{8}$ As most patients were in the USA, findings may not apply in other locations; it was not possible to analyse separately the $7 \%$ of patients from other countries. Nationwide studies and prospective cohort studies in different settings are needed to complement these findings, and to assess how the incidence trajectory changes as the pandemic progresses. It is possible that the incidence may continue to rise further if there remains an excess of individuals in the community yet to present or receive a diagnosis; alternatively, the incidence might fall if some of the recent excess reflects people whose diagnosis was made earlier than would have otherwise been the case.

In summary, our findings support the view that the incidence and outcomes of eating disorders have been adversely affected by the COVID-19 pandemic. $^{1-4,9-11}$ Services need to be resourced adequately and urgently to deal with the increased demand.

Maxime Taquet, Department of Psychiatry, University of Oxford, Warneford Hospital UK; and Oxford Health NHS Foundation Trust, UK; John R. Geddes, Department of Psychiatry, University of Oxford, Warneford Hospital, UK; and Oxford Health NHS

Foundation Trust, UK: Sierra Luciano, TriNetX, USA; Paul J. Harrison (D), Department of Psychiatry, University of Oxford, Warneford Hospital, UK; and Oxford Health NHS Foundation Trust, UK

Correspondence: Paul J. Harrison. Email: paul.harrison@psych.ox.ac.uk

First received 12 May 2021, final revision 24 Jun 2021, accepted 25 Jun 2021

\section{Supplementary material}

To view supplementary material for this article, please visit https://doi.org/10.1192/bjp.2021. 105 


\section{Data availability}

The TriNetX system returned the results of these analyses as csv files that were downloaded and archived. Data presented in this paper can be accessed at https://osf.io/nukz4. Additionally, TrinetX will grant access to researchers if they have a specific concern (via the third-party agreement option).

\section{Author contributions}

M.T. and P.J.H. designed the study. M.T. conducted the analyses, assisted by S.L. M.T. and P.J.H. wrote the paper with input from J.R.G. and S.L.. All authors approved the submission.

\section{Funding}

Supported by the National Institute for Health Research (NIHR) Oxford Health Biomedical Research Suppre (BRC) MT is an NHR Academic Clinical Fellow and an N Research Fellow. S.L. is an employee of TrinetX. The views expressed are those of the authors and not necessarily those of the National Health Service, NIHR or the Department of Health.

\section{Declaration of interest}

M.T. and P.J.H. were granted unrestricted access to the TriNetX Analytics network for the purpose of research relevant to psychiatry and with no constraints on the analyses performed no the decision to publish. S.L. is an employee of TriNetX. J.R.G., a member of the Editorial Board, played no part in the review or decision-making process of this paper.

\section{References}

1 Touyz S, Lacey H, Hay P. Eating disorders in the time of COVID-19. J Eat Disord 2020; 8: 19 .

2 Rodgers RF, Lombardo C, Cerolini S, Franko DL, Omori M, Fuller-Tyszkiewicz M, et al. The impact of the COVID-19 pandemic on eating disorder risk and symptoms. Int J Eat Disord 2020; 53: 1166-70.
3 Haripersad YV, Kannegiesser-Bailey M, Morton K, Skeldon S, Shipton N Edwards $\mathrm{K}$, et al. Outbreak of anorexia nervosa admissions during the COVID-19 pandemic. Arch Dis Child 2021; 106: e15.

4 Solmi F, Downs JL, Nicholls DE. COVID-19 and eating disorders in young people. Lancet Child Adolesc Health 2021; 5: 316-8.

5 Taquet M, Geddes JR, Husain M, Luciano S, Harrison PJ. 6-month neurological and psychiatric outcomes in 236379 survivors of COVID-19: a retrospective cohort study using electronic health records. Lancet Psychiatry 2021; 8 : $416-27$.

6 World Health Organization. ICD-10 International Statistical Classification of Diseases and Related Health Problems, 10th Revision. WHO, 2019.

7 Taquet M, Luciano S, Geddes JR, Harrison PJ. Bidirectional associations between COVID-19 and psychiatric disorder: retrospective cohort studies of 62354 COVID-19 cases in the USA. Lancet Psychiatry 2021; 8: $130-40$.

8 Hart A, Granillo MT, Jorm AF, Paxton SJ. Unmet need for treatment in the eating disorders: a systematic review of eating disorder specific treatment seeking among community cases. Clin Psychol Rev 2011; 31: 727-35.

9 Monteleone AM, Marciello F, Cascino G, Abbate-Daga G, Anselmetti S, Baiano M et al. The impact of COVID-19 lockdown and of the following "re-opening" period on specific and general psychopathology in people with Eating Disorders: the emergent role of internalizing symptoms. J Affect Disord 2021; 285: 77-83.

10 Castellini G, Cassioli E, Rossi E, Innocenti M, Gironi V, Sanfilippo G, et al. The impact of COVID-19 epidemic on eating disorders: a longitudinal observation of pre versus post psychopathological features in a sample of patients with eating disorders and a group of healthy controls. Int J Eat Disord 2020; 53 : 1855-62.

11 Spettigue W, Obeid N, Erbach M, Feder S, Finner N, Harrison ME, et al. The impact of COVID-19 on adolescents with eating disorders: a cohort study. J Eat Disord 2021; 9: 65 